\title{
Spin echo functional MRI in bilateral auditory cortices at 7T: An application of $B(1)$ shimming
}

Citation for published version (APA):

de Martino, F., Schmitter, S., Moerel, M., Tian, J., Ugurbil, K., Formisano, E., Yacoub, E., \& van de Moortele, P. F. (2012). Spin echo functional MRI in bilateral auditory cortices at 7T: An application of B(1) shimming. Neuroimage, 63(3), 1313-1320. https://doi.org/10.1016/j.neuroimage.2012.08.029

Document status and date:

Published: 15/11/2012

DOI:

10.1016/j.neuroimage.2012.08.029

Document Version:

Publisher's PDF, also known as Version of record

Document license:

Taverne

Please check the document version of this publication:

- A submitted manuscript is the version of the article upon submission and before peer-review. There can be important differences between the submitted version and the official published version of record.

People interested in the research are advised to contact the author for the final version of the publication, or visit the DOI to the publisher's website.

- The final author version and the galley proof are versions of the publication after peer review.

- The final published version features the final layout of the paper including the volume, issue and page numbers.

Link to publication

\footnotetext{
General rights rights.

- You may freely distribute the URL identifying the publication in the public portal. please follow below link for the End User Agreement:

www.umlib.nl/taverne-license

Take down policy

If you believe that this document breaches copyright please contact us at:

repository@maastrichtuniversity.nl

providing details and we will investigate your claim.
}

Copyright and moral rights for the publications made accessible in the public portal are retained by the authors and/or other copyright owners and it is a condition of accessing publications that users recognise and abide by the legal requirements associated with these

- Users may download and print one copy of any publication from the public portal for the purpose of private study or research.

- You may not further distribute the material or use it for any profit-making activity or commercial gain

If the publication is distributed under the terms of Article $25 \mathrm{fa}$ of the Dutch Copyright Act, indicated by the "Taverne" license above, 


\title{
Spin echo functional MRI in bilateral auditory cortices at $7 \mathrm{~T}$ : An application of $\mathrm{B}_{1}$ shimming
}

\author{
Federico De Martino a,b,c,*,1, Sebastian Schmitter ${ }^{\text {a, }}{ }^{\text {, }}$, Michelle Moerel ${ }^{\text {b,c }}$, Jinfeng Tian ${ }^{\text {a }}$, Kamil Ugurbil ${ }^{\text {a }}$, \\ Elia Formisano ${ }^{b, c}$, Essa Yacoub ${ }^{a}$, Pierre-Francois van de Moortele ${ }^{a}$ \\ a Center for Magnetic Resonance Research, University of Minnesota Medical School, Minneapolis, MN 55455, USA \\ ${ }^{\mathrm{b}}$ Department of Cognitive Neuroscience, Faculty of Psychology and Neuroscience, Maastricht University, 6200MD Maastricht, The Netherlands \\ ${ }^{c}$ Maastricht Brain Imaging Center (M-BIC), Maastricht University, 6200MD Maastricht, The Netherlands
}

\section{A R T I C L E I N F O}

\section{Article history:}

Accepted 11 August 2012

Available online 21 August 2012

\section{Keywords:}

$7 \mathrm{~T}$

BOLD

fMRI

Spin echo

$\mathrm{B}_{1}$ shimming

Auditory cortex

\begin{abstract}
A B S T R A C T
Ultra high fields (UHF) permit unprecedented explorations of functional organizations and insight into basic neuronal processes. Increases in the signal and contrast to noise ratios have allowed increases in the spatial resolution of $T_{2}^{*}$ weighted gradient echo (GE) echo planar imaging (EPI). Furthermore, while the use of $T_{2}$ weighted imaging methods at UHF (e.g. spin echo (SE) EPI, gradient and spin echo (GRASE) EPI) can also permit higher resolution images, they in addition allow for increased spatial specificity of functional responses, permitting the in-vivo study of functional organizations down to the columnar level of the cortex.

The study of the visual cortex has, thus far, benefitted the most from higher resolution $\mathrm{T}_{2}$ weighted studies as achieving the required transmit $\mathrm{B}_{1}$ magnitude at $7 \mathrm{~T}$ is more challenging in other brain regions, such as the auditory cortex. As such, auditory fMRI studies at UHF have been limited to $\mathrm{T}_{2}{ }^{*}$ weighted GE sequences.

Recent advances in multi-channel RF transmission (e.g. $B_{1}$ shimming) have enabled procedures to efficiently address deficiencies in transmit $\mathrm{B}_{1}$ profiles. However, these techniques, shown to be advantageous in anatomical imaging at UHF, are not generally utilized to facilitate $\mathrm{T}_{2}$ weighted fMRI studies.

Here we investigate the feasibility of applying $B_{1}$ shimming to achieve efficient $R F$ transmission in the human auditory cortex. We demonstrate that, with $\mathrm{B}_{1}$ shimming, functional responses to simple tones and to complex sounds (i.e. voices, speech, animal cries, tools and nature) can be efficiently measured with $\mathrm{T}_{2}$ weighted SE-EPI in the bilateral human auditory cortex at $7 \mathrm{~T}$ without exceeding specific absorption rate (SAR) limits.
\end{abstract}

(c) 2012 Elsevier Inc. All rights reserved.

\section{Introduction}

With the introduction of functional MRI ( $\mathrm{FMRI}$ ) relying on the blood oxygenation level dependent (BOLD) mechanism (Bandettini et al., 1992; Kwong et al., 1992; Ogawa et al., 1992), in-vivo MR studies of brain structure have been complemented with studies of brain function. BOLD fMRI has proven to be a highly attractive technique due to its relatively high spatial resolution, the absence of exogenous contrast agents and ionizing radiations, and because of the widespread access to MR scanners. This, along with hardware and software developments that have permitted ultrafast and efficient acquisitions, has rapidly made fMRI the method of choice for in vivo functional studies of the human brain.

\footnotetext{
* Corresponding Author at: Department of Cognitive Neurosciences, Faculty of Psychology, University of Maastricht, The Netherlands, Postbus 616, 6200 MD, Maastricht, The Netherlands. Tel.: + 3143 3884532; Fax: + 31433884125.

E-mail address: f.demartino@maastrichtuniversity.nl (F. De Martino).

1 These authors contributed equally to this work.
}

It has been demonstrated that operating at higher magnetic fields, as opposed to conventional clinical field strengths (3 $\mathrm{T}$ and lower), offers substantial improvements for functional and anatomical studies (Vaughan et al., 2001; Yacoub et al., 2001; Ugurbil et al., 2003). For functional MRI studies, the benefits of UHF are several-fold. First, the intrinsic signal to noise ratio is approximately proportional to the main $\mathrm{B}_{0}$ field, permitting the acquisition of higher spatial resolution images. Whereas the majority of functional images at clinical field strength ( $3 \mathrm{~T}$ and below) are acquired with a spatial resolution of about $27 \mathrm{~mm}^{3}$, data can be acquired at UHF with significantly higher resolutions $\left(1-3.7 \mathrm{~mm}^{3}\right)$ without losses in temporal signal-to-noise (tSNR) and thus without losses in the sensitivity to activated regions (Ugurbil et al., 2003). Second, while the extravascular BOLD contribution of large draining veins increases linearly with $\mathrm{B}_{0}$ due to static averaging effects, contributions from smaller vessels, which are more closely correlated with neuronal activity, increase quadratically with $\mathrm{B}_{0}$ due to dynamic averaging effects (Ogawa et al., 1992; Uğurbil et al., 2003, 2006). Furthermore, at UHF, the transverse relaxation time constants of blood $\left(\mathrm{T}_{2}^{*}\right.$ and $\mathrm{T}_{2}$ ) are much shorter than that of brain tissues, resulting in a smaller blood contribution (intravascular BOLD) to the 
fMRI signal. As a result, while at clinical field strengths BOLD activations are dominated by large draining veins, at UHF smaller vessels contribute more to the measured activation. This decreases the width of the spatial point-spread-function (PSF) of the activation (Shmuel et al., 2007), strengthening its link to the underlying neuronal activity. Lastly, only at UHFs has the use of $T_{2}$ instead of $T_{2}^{*}$ weighted BOLD images for fMRI proven to be beneficial. This is because the BOLD response from areas surrounding large veins is largely reduced in $T_{2}$ weighted images at UHF, while the contribution from extra-vascular compartments around capillaries or small vessels grows substantially (Lee et al., 1999; Duong et al., 2003; Yacoub et al., 2003, 2005). The aforementioned advantages of $\mathrm{T}_{2}$ weighted fMRI at UHF have been applied to the mapping of columnar organizations in the human visual cortex (Yacoub et al., 2007, 2008; Zimmermann et al., 2011).

In the auditory cortex we previously demonstrated, for the first time in humans, the mirror symmetric organization of tonotopic maps using $\mathrm{T}_{2}^{*}$ weighted fMRI at $7 \mathrm{~T}$ (Formisano et al., 2003). At this time, multi-channel coil technology was not available. Therefore, in order to achieve sufficient transmit and receive $B_{1}$ fields in the auditory cortex, a local single channel surface RF coil along with a zoomed GE-EPI fMRI acquisition was used providing the necessary resolution and sensitivity for auditory fMRI studies (Formisano et al., 2003). In the current work, the goal was to use recent advances in multi-channel coil technology to achieve the necessary sensitivity and efficiency for $T_{2}$-weighted fMRI of the auditory cortex at $7 \mathrm{~T}$. Until now, application of SE-EPI to sensory areas other than the visual one has been used in only a few studies (Harmer et al., 2011), and high resolution/columnar level studies, that directly take advantage of $\mathrm{T}_{2}$ weighted functional imaging (Norris, 2012), have been limited to visual areas (Yacoub et al., 2007, 2008). Further, while the large-scale functional organization of the auditory cortex has been extensively studied with GE-EPI (Belin et al., 2000; Formisano et al., 2003; Schönwiesner and Zatorre, 2009; Da Costa et al., 2011), no $\mathrm{T}_{2}$ weighted functional study has been conducted in this primary sensory region. Several constraints to the use of $T_{2}$ weighted functional imaging outside of visual areas can be highlighted. First, since $\mathrm{T}_{2}$ weighted fMRI relies on the use of $180^{\circ}$ refocusing RF pulses, which necessitate higher local transmit $\mathrm{B}_{1}$ and thus higher RF power, its use at $7 \mathrm{~T}$ can be rapidly limited by the specific absorption rate (SAR). While this problem can be efficiently solved by designing surface or surface array coils with high local transmit $B_{1}$ fields over the cortical areas of interest, this approach, however, necessarily sacrifices sensitivity to more distant cortical and sub-cortical areas, other sensory areas, and whole brain anatomical imaging. Further, it would require separate coils to achieve bi-lateral coverage or to study different areas. On the other hand, conventional $7 \mathrm{~T}$ volume coils are characterized by a transmit $B_{1}$ profile which is high in the center of the brain, but rapidly decays towards the periphery, unfortunately resulting in poor RF efficiency and non-uniformity in the auditory cortices. Using such coils for $\mathrm{T}_{2}$ weighted auditory fMRI would require large increases in the transmit voltage in order to achieve sufficient refocusing flip angles in auditory cortices. The increase in power does not guarantee uniform refocusing in these areas, and, furthermore, imposes the use of either a long repetition time and/or a very limited number of slices to not exceed SAR limits.

Advances in parallel excitation technology, including multi-channel transmit RF coils at $7 \mathrm{~T}$ (Adriany et al., 2005, 2008) and $\mathrm{B}_{1}$ shimming methods, provide a means by which transmit fields at UHF can be shaped, promising to extend high resolution SE-fMRI applications to other brain regions. In particular, fast multi-channel $B_{1}$ calibration (van de Moortele et al., 2007; van de Moortele and Ugurbil, 2009) can be used to adjust the phases of multiple transmit channels in order to achieve maximum transmit $B_{1}$ efficiency in one or several regions of interest chosen by the operator, as was demonstrated in the brain and in the torso at 7 T (van De Moortele et al., 2005; Bandettini et al., 2011; Metzger et al., 2011; Ellermann et al., 2012; Schmitter et al., 2012). Here, we apply similar $B_{1}$ shimming methods with a multi-channel
$7 \mathrm{~T}$ whole head coil in order to demonstrate the feasibility of obtaining multi-slice $\mathrm{T}_{2}$ weighted SE-EPI fMRI in bilateral auditory cortices. As a case study we measured functional responses to both simple tones and complex natural sounds (e.g. voices, speech, animal cries, tools, nature) in an attempt to map both tonotopy and voice selective regions in the human auditory cortex.

\section{Methods}

\section{Ethics statement}

Five subjects ( 4 males and 1 female with normal hearing), who signed a written informed consent form, were enrolled in this $7 \mathrm{~T}$ study approved by the institutional review board for human subject research at the University of Minnesota

\section{Experimental design and stimuli}

Auditory stimuli were presented using high quality MRI compatible insert earphones with a flat response over [0-8 kHz] bandwidth (Sensimetrics Corporation, USA). Subjects were instructed to fixate on a small central fixation spot during the entire functional scan and passively listen. Stimuli consisted of $800 \mathrm{~ms}$ long amplitude-modulated ( $8 \mathrm{~Hz}$; modulation depth of 1 ) tones $(.45 ; .5 ; .55 ; 1.35 ; 1.5 ; 1.65 ; 2.25$; $2.5 ; 2.75 \mathrm{kHz})$ grouped in blocks around three center frequencies (.5; $.1 .5 ; 2.5 \mathrm{kHz}$ ) together with blocks of natural sounds ( $800 \mathrm{~ms}$ recordings) grouped in blocks of five different categories (speech, voice, animals, tools, nature). Sounds were equalized in RMS and their on- and offsets were ramped with a $10 \mathrm{~ms}$ linear slope. Each block consisted of six sounds, lasted $18 \mathrm{~s}$, and was separated by $12 \mathrm{~s}$ of silence from the following block. Each condition was presented twice per run, with six runs acquired per subject. All stimuli were presented in silent gaps in between the acquisition of each brain volume.

\section{MRI acquisition}

Data acquisition was performed at the Center for Magnetic Resonance Research (Minneapolis, MN, USA) using a $90 \mathrm{~cm}$ bore $7 \mathrm{~T}$ whole-body magnet (Magnex Scientific, Abingdon, UK) driven by a Siemens console (Siemens Medical Systems, Erlangen, Germany). A custom 8 transmit/receive 24 receive-only channel RF coil powered by eight $1 \mathrm{~kW}$ RF amplifiers (CPC, USA) was used for all data acquisition sessions. A phase and gain controller unit (CPC, USA) allowed setting the phase and amplitude of the low voltage input of each of the $8 \mathrm{RF}$ amplifiers remotely (from an external PC through Ethernet communication), after $B_{1}$ shimming computations. For patient safety, in addition to standard MR console RF monitoring operating on one channel, an in-house developed system continuously monitored RF power output for each RF amplifier over two averaging time windows $(10 \mathrm{~s}$ and $600 \mathrm{~s}$ ). All RF amplifiers are immediately shut down should RF power exceed maximum allowed limits on any single channel. Details on this $\mathrm{RF}$ monitoring setup can be found in Metzger et al. (2010). In order to not exceed SAR limits as defined by IEC 60601-2-33, 3rd edition, we performed electromagnetic simulations at $300 \mathrm{MHz}$ with a finite difference time domain solver (XFDTF, Remcom, Pittsburg, PA) based on the design of the 8 channel transmit RF coil loaded with the model of a human head. We obtained complex $B_{1}$ and $E$ field maps for each of the 8 transmit elements, and we calculated $B_{1}$ shimming solution over the auditory cortices in the head model with the same $B_{1}$ shimming algorithm used in-vivo. We then applied the complex coefficients of this solution to the electric field maps to obtain the resulting 3D electric field map and we computed global and local $10 \mathrm{~g}$ average SAR maps. We utilized the ratio of the highest local $10 \mathrm{~g}$ average SAR value over global SAR to determine the maximum global SAR limit for our in-vivo experiments in order to keep within IEC 60601-2-33 
guidelines (i.e. $3.2 \mathrm{~W} / \mathrm{kg}$ volume head and $20 \mathrm{~W} / \mathrm{kg} 10 \mathrm{~g}$ average local transmit coils in head in first controlled mode).

Each session started with a set of eight transmit $B_{1}$ phases applied to the $B_{1}$ shim unit to obtain a spatial $B_{1}$ pattern similar to a volume coil by approximating a circularly polarized (CP-like) mode (Schmitter et al., 2012). With this $B_{1}$ shimming setting, a scout localizer was acquired, followed by a 3D $\mathrm{T}_{1}$ weighted MPRAGE anatomical data set ( $1 \mathrm{~mm}$ isotropic resolution) used for positioning subsequent acquisitions. Complex $\mathrm{B}_{1}^{+}$maps of the 8 transmit channels were obtained by a fast $\mathrm{B}_{1}^{+}$ calibration in the small flip angle regime in an oblique slice crossing both auditory cortices (van de Moortele and Ugurbil, 2009). A B $B_{1}$ shimming solution, consisting of a specific RF phase for each transmit channel, was calculated to maximize $B_{1}$ efficiency by reducing destructive interferences within two regions of interest manually drawn over bilateral auditory areas (Ellermann et al., 2012; Metzger et al., 2012). The mean and the coefficient of variation $(\mathrm{CV})$, with $\mathrm{CV}=$ mean $\div$ std, of relative transmit $B_{1}$ efficiency (defined as the ratio $\left|\Sigma\left(B_{1, k}^{+}\right)\right| \div \Sigma\left(\left|B_{1, k}^{+}\right|\right.$, with $\mathrm{k}=1, \ldots, 8)$ and excitation flip angle were computed inside the ROIs before and after $\mathrm{B}_{1}$ shimming. The RF power was calibrated accordingly based on a 3D AFI map acquired after applying the computed $\mathrm{B}_{1}$ shimming solution (Yarnykh, 2007). Multi-slice $\mathrm{T}_{2}$ weighted SE-EPI images were collected before (one volume) and after (fMRI series) $B_{1}$ shimming to demonstrate the efficacy of the method. A standard 2D SE-EPI sequence (27 transverse oblique slices; $\mathrm{TE}=37 \mathrm{~ms}$; IPAT $=3$, $128 \times 128,1.5 \times 1.5 \times 1.5 \mathrm{~mm}^{3} ; \mathrm{TR}=3 \mathrm{~s}$; gap $=1.5 \mathrm{~s}$ ) was used for all measurements. All $\mathrm{B}_{1}$-related computations were performed in Matlab (MathWorks, Natick, MA).

Since subjects were taken from a pool of subjects that had previously participated in a $3 \mathrm{~T}$ (Skyra, Siemens Medical Systems, Erlangen, Germany) study, anatomical images (3D $\mathrm{T}_{1}$ weighted MPRAGE; $1 \mathrm{~mm}$ isotropic) from the $3 \mathrm{~T}$ session were used for whole brain segmentation and white/gray matter boundary identification.

\section{fMRI data analysis}

Functional and anatomical images were analyzed using BrainVoyager QX (Brain Innovation, Maastricht, The Netherlands).

Preprocessing of the functional data included: inter-scan slice-time correction, 3D rigid body motion correction, moderate spatial smoothing ( $2 \mathrm{~mm}$ full width half maximum [FWHM]), high-pass filtering using a general linear model (GLM) Fourier basis set and a temporal Gaussian smoothing with a FWHM kernel of 2 data-points. Functional runs were co-registered to the individual anatomical $\mathrm{T}_{1}$ scan acquired in the same session using the scanners' positional information followed by boundary based registration. Anatomical data were aligned across sessions to allow for co-registration of functional data to the $\mathrm{T}_{1}$-weighted images. The anatomical data were projected in Talairach space and automatically segmented. After obtaining single subject surfaces of both left and right hemispheres, cortex based alignment (Goebel et al., 2006) was used to obtain average surfaces across the five subjects. Finally, the functional data were sinc-interpolated to 3D Talairach space with a resolution of $1 \mathrm{~mm}$ isotropic.

All statistical computations were performed on a single-subject and group (fixed effects) level using a GLM with a linear predictor for each experimental condition convolved with a standard hemodynamic response function. Tonotopic maps were obtained for each subject by best frequency mapping (Formisano et al., 2003) using the estimated beta values for the three different frequency blocks (high, middle and low frequencies). Single subject maps were limited to voxels exhibiting significant $(q[F D R]=0.05)$ responses to all sounds at the group level. Group tonotopic maps were obtained by averaging single subject maps in the cortex-based aligned space and where limited to vertices present in at least three of the five subjects. Speech and voice selective regions were obtained by contrasting speech/voice responses with responses to all other natural sounds (tools, animal cries and nature sounds). Single subject and group maps (fixed effects General Linear Model) were corrected for multiple comparisons using false discovery rate (Genovese et al., 2002) and cluster level based correction (Forman et al., 1995).

\section{Results}

In each subject, $B_{1}$ shimming significantly increased relative $B_{1}$ efficiency over the bilateral auditory cortex, as summarized in Table 1, which shows the mean and coefficient of variation of relative $\mathrm{B}_{1}$ efficiency in all subjects, before and after $\mathrm{B}_{1}$ shimming. Averaged over all subjects, the efficiency increases from 0.43 before shimming to 0.78 after shimming. Correspondingly, the flip angle within the auditory cortices increased significantly while keeping the same transmitter voltage before and after $\mathrm{B}_{1}$ shimming (note that no $\mathrm{B}_{1}^{+}$ map before shimming was available for subject 2). Averaged over 4 of 5 subjects, the mean flip angle increased from $42.5^{\circ}$ to $92.8^{\circ}$ for a nominal target flip angle of $90^{\circ}$, while using identical $\mathrm{RF}$ power input. For illustration, $B_{1}$ efficiency and flip angle maps are shown in Fig. 1 for subject 1 before and after $B_{1}$ shimming on the oblique slice utilized for $\mathrm{B}_{1}$ shimming computation, with the ROI overlaid on the corresponding maps. As expected the resulting spatial $\mathrm{B}_{1}$ pattern after shimming shows stronger $\left|\mathrm{B}_{1}^{+}\right|$amplitude in the ROIs, to the cost of very low $\left|\mathrm{B}_{1}^{+}\right|$values, resulting from destructive interference, in a limited area at the center of the brain, which was deliberately not included in the optimization ROIs. This pattern was consistently observed in all subjects. The significant reduction in required RF power for a given target flip angle, with $\mathrm{B}_{1}$ shimming, made it possible to acquire 27 SE-EPI slices with a $3 \mathrm{~s}$ TR without exceeding maximum SAR limits. On the contrary, without $B_{1}$ shimming but with voltage adjustment to achieve the target flip angle $\left(90^{\circ}\right)$ in the bilateral auditory cortex, only about 9 slices could have been sampled in the same TR in subject 1 due to SAR limitations. Furthermore, in subject \#4 the target flip angle could not even been achieved in the auditory cortex without $B_{1}$ shimming since the necessary RF peak power exceeded the maximum capability of our transmit RF chain. Interestingly, as shown in Table 1, the coefficient of variation of the measured flip angle consistently dropped inside the ROI after $\mathrm{B}_{1}$ shimming, from 0.22 to 0.34 down to $0.10-0.14$, even though the parameter maximized in our $B_{1}$ shimming algorithm was the average relative $B_{1}$ efficiency, an observation fully consistent with a previous report on $B_{1}$ shimming in the prostate at 7 T where increasing transmit $B_{1}$ phase coherence inside a relatively small anatomical target also resulted in a net increase in flip angle average magnitude and homogeneity inside the ROI (Metzger et al., 2008).

As expected, given the typical cubic dependency of SE image intensity on $\left|\mathrm{B}_{1}^{+}\right|$(Stollberger and Wach, 1996), maximizing $\mathrm{B}_{1}$ efficiency resulted in substantial improvement in SE-EPI image quality. This is illustrated for two subjects in Fig. 2, showing SE-EPI images

Table 1

Single subject $\mathrm{B}_{1}$ shimming results.

\begin{tabular}{lllllllll}
\hline \multirow{2}{*}{$\begin{array}{l}\text { Measured parameter } \\
\text { within ROI }\end{array}$} & $\begin{array}{l}\mathrm{B}_{1} \text { shimming } \\
\text { status }\end{array}$ & & \multicolumn{3}{l}{ Subject } \\
\cline { 4 - 9 } & & & 1 & 2 & 3 & 4 & 5 \\
\hline $\mathrm{B}_{1}$ relative efficiency & Before & Mean & 0.47 & 0.35 & 0.44 & 0.39 & 0.49 \\
& & CV & 0.55 & 0.26 & 0.30 & 0.28 & 0.29 \\
& After & Mean & 0.78 & 0.76 & 0.81 & 0.76 & 0.76 \\
& & CV & 0.23 & 0.25 & 0.27 & 0.29 & 0.28 \\
Excitation flip angle & Before & Mean & 54.6 & n/a & 47.7 & 31.5 & 36.2 \\
(target $=90^{\circ}$ ) & & CV & 0.22 & n/a & 0.34 & 0.27 & 0.30 \\
& After & Mean & 96.3 & 97.0 & 91.2 & 88.7 & 95.1 \\
& & CV & 0.10 & 0.11 & 0.12 & 0.14 & 0.10
\end{tabular}

$B_{1}$ relative efficiency and excitation flip angles measured within two bilateral auditory cortex ROIs. Mean and coefficient of variation across all voxels in the ROIs are reported before and after $\mathrm{B}_{1}$ shimming. 
Before B1 shimming
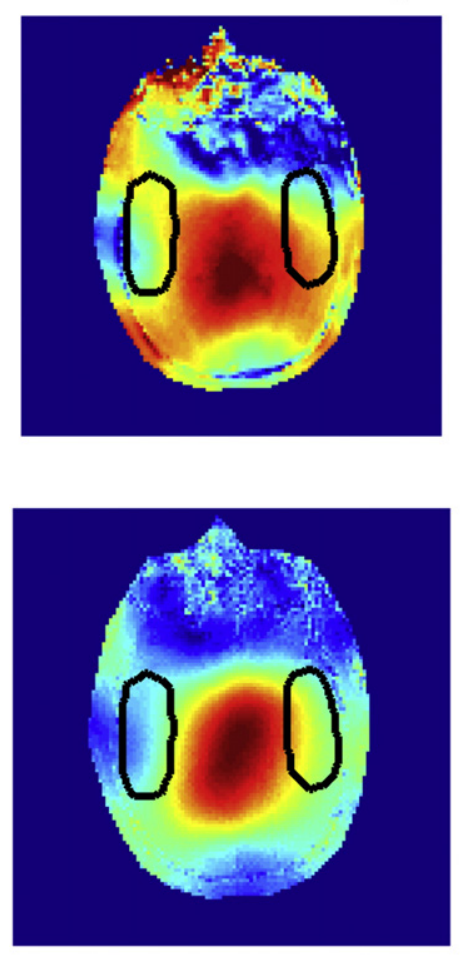

After B1 shimming
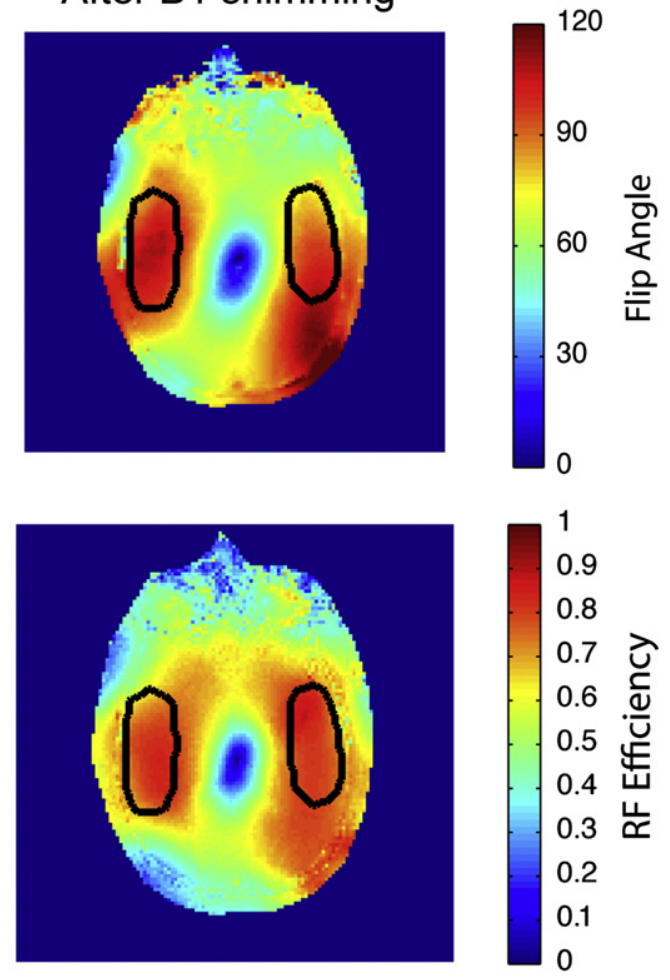

Fig. 1. $B_{1}$ relative efficiency and flip angle maps before and after $B_{1}$ shimming in one subject. The targeted bilateral auditory cortex ROIs are outlined in black.

acquired before and after $B_{1}$ shimming. Although initial $\left|B_{1}^{+}\right|$profiles were not identical in all subjects before $B_{1}$ shimming, similar SE-EPI image quality was obtained in all experiments after $B_{1}$ shimming, without apparent dependency on pre-shimming $\left|\mathrm{B}_{1}^{+}\right|$patterns. Also, as illustrated in Fig. 2, significant (FDR corrected) responses to sounds could be detected bilaterally in each subject after $\mathrm{B}_{1}$ shimming (given the low quality of corresponding SE-EPI images, no attempts were made to collect SE fMRI time series before $\mathrm{B}_{1}$ shimming).

In each subject, voice selective regions were identified and reliable tonotopy maps (i.e. frequency selectivity) were obtained, as illustrated in one subject in Fig. 3. Tonotopic maps (high frequencies color coded in blue and low frequencies color coded in red) and voice selective areas $(q[F D R]=0.05$; t-values color coded from red to yellow) are projected on the individual subject surface reconstruction of the left and right hemisphere. Note the high-low-high mirror symmetric gradient in the tonotopic maps over the bilateral Heschl's region (Formisano et al., 2003) and the bilateral selectivity to voices of regions along the superior temporal sulcus (STS) and gyrus (STG) (Belin et al., 2000).

Group $(\mathrm{N}=5)$ results for tonotopy and voice selective regions (cluster level corrected $\mathrm{p}=0.001$ ) are reported in Fig. 4. Functional maps are projected on the mean surface of left and right hemispheres obtained after cortex-based alignment (first three rows). Voice selective regions are also presented superimposed to four transversal slices of the mean anatomical data after Talairach alignment.

\section{Discussion}

Functional MRI has enabled human studies at high spatial resolutions and specificity (Menon et al., 1997; Cheng et al., 2001; Sun et al., 2007; Yacoub et al., 2007, 2008; Zimmermann et al., 2011), permitting unprecedented explorations of functional organizations and insights into basic neuronal processes, with higher field studies leading the way (Yacoub et al., 2001; Ugurbil et al., 2003). However, due to the many technical issues incurred at higher fields, the vast majority of these studies have been conducted with surface coils and in visual areas, which are more easily accessible than other brain regions. Extending high resolution high specificity acquisitions to other areas requires either local surface coils for each area of interest, or multi-channel coil technology in combination with whole head coils which could allow for imaging of multiple sensory areas, bi-lateral sensitivity to sensory areas, sensitivity to sub-cortical areas, and whole brain anatomical imaging with the same coil. Here, we demonstrate the feasibility of using a multi-channel whole head coil to obtain sufficient $\mathrm{B}_{1}$ efficiency in bilateral auditory cortices for SE-based fMRI acquisitions. fMRI cortical responses to synthetic (e.g. tones) and complex natural (e.g. voices, speech) sounds have previously only been investigated (Belin et al., 2000; Formisano et al., 2003; Schönwiesner and Zatorre, 2009; Da Costa et al., 2011; Striem-Amit et al., 2011) with conventional GE echo-planar imaging. In the case of human auditory functional studies, the use of the more specific $\mathrm{T}_{2}$-weighted functional contrast mechanism at high fields (Yacoub et al., 2003, 2005) has been precluded by the challenge of achieving efficient transmit fields over bilateral or even in a single hemisphere in auditory areas with conventional volume transmission coils, and by the lack of surface array coils designed for auditory investigations.

Here, we combined $\mathrm{B}_{1}$-shimming and SE-fMRI at $7 \mathrm{~T}$ to measure functional responses to synthetic ("tones") and complex natural sounds. Making use of parallel transmission capabilities (i.e. multiple amplifiers and a volume coil with multiple transmit elements), $B_{1}$-shimming shapes the transmit fields in order to reduce destructive interference (van De Moortele et al., 2005) and achieve maximum transmit efficiency in selected region(s) of interest, and has been successfully employed at $7 \mathrm{~T}$ in a variety of anatomical targets (Metzger et al., 2008; Ellermann et al., 2012). Our results demonstrate that by adjusting the phases of the transmit elements $(C=8)$ we were able to significantly increase transmit $B_{1}$ efficiency over the bilateral auditory cortex (see Fig. 1) by 
a)

\section{Before B1 shimming}

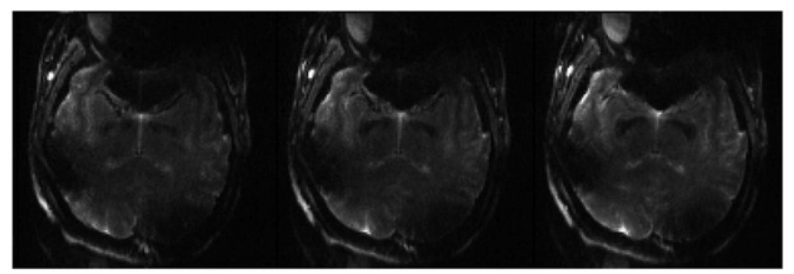

b)

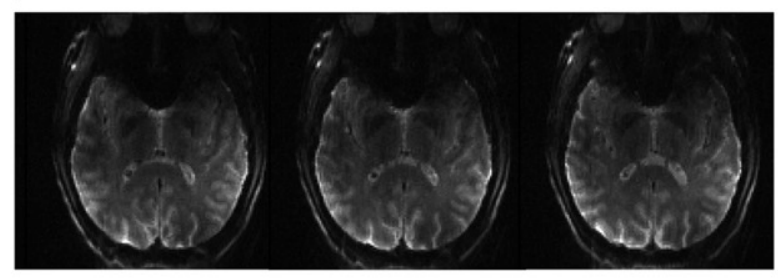

After B1 shimming

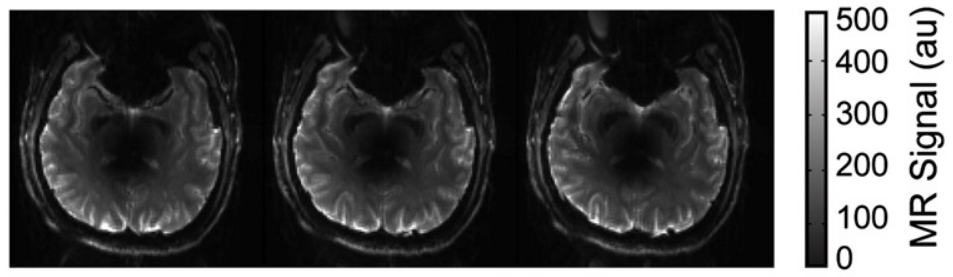

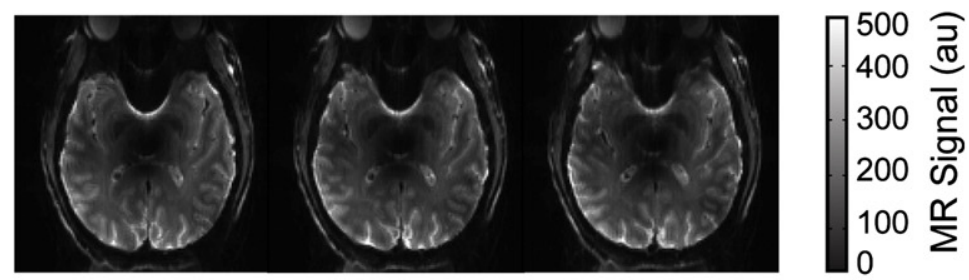

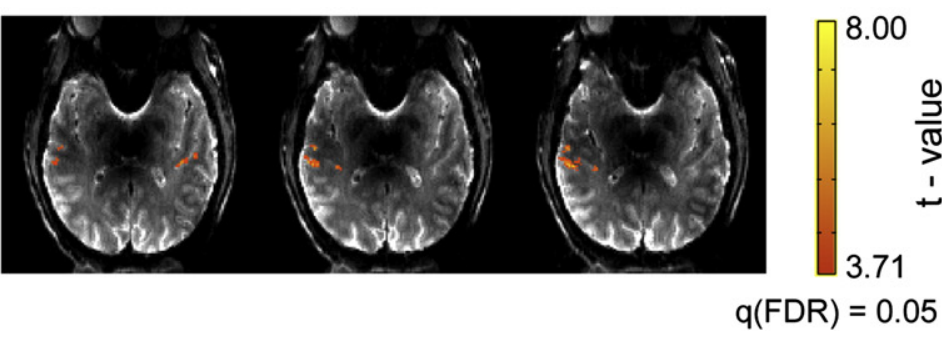

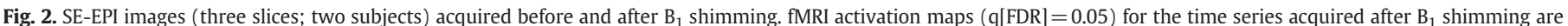
reported in the same slices.

more than a factor of 1.8. The improved transmit $B_{1}$ efficiency allowed measuring 27 slices at $1.5 \mathrm{~mm}$ isotropic resolution with a repetition time of $3 \mathrm{~s}$ without exceeding SAR limits. This represents a three-fold improvement with respect to the coverage obtainable without $B_{1}$ shimming ( 9 slices at $1.5 \mathrm{~mm}$ isotropic resolution with the same TR of $3 \mathrm{~s}$ ) in volunteer 1 . In addition to SAR guidelines, the maximum available RF peak power can also be a hard limit on protocols. With our hardware configurations, the required voltage to attain the target flip angles $\left(90^{\circ}\right.$ excitation and $180^{\circ}$ refocusing RF pulses) could not be achieved in some volunteers, without $\mathrm{B}_{1}$ shimming, due to limitations in RF peak power. The improved brain coverage allowed measuring auditory cortical areas from the inferior temporal sulcus (ITS) to the planum temporale, de facto covering the whole temporal cortex bilaterally. The shear improvement in SE-EPI image quality in the auditory cortices was accompanied by expected drops of sensitivity in neglected regions such as the thalamus (see Fig. 2). Although these regions were deliberately not included in the present study, more sophisticated use of parallel transmit methods could allow for extending areas with satisfactory refocusing RF pulses, should such areas be a concomitant interest in further studies. It is noteworthy that adiabatic pulse could be used to obtain a homogeneous distribution of flip angles (due to the insensitivity of adiabatic pulses to the $B_{1}$ profile above adiabatic threshold). However, in order to achieve phase coherence with slice selective adiabatic inversion, two adiabatic refocusing pulses are typically used after each excitation pulse. For same SAR per pulse, this would result in roughly a maximum of twice fewer slices, even without considering the likelihood that each adiabatic inversion pulse yields higher SAR than standard sinc inversion pulses. Furthermore, the required peak power for adiabatic inversion may exceed RF amplifier capability. Considering our protocol, using adiabatic refocusing would require dropping from 9 slices for standard spin-echo without $B_{1}$ shim down to 4 or 5 slices. This is to be compared with 27 slices for standard spin echo with $B_{1}$ shim.

By measuring high quality SE-fMRI images, we were able to map tonotopic responses and voice selective regions in single subjects $(\mathrm{N}=$ 5). In each individual subject and in the group, the topographic arrangement of frequency selectivity was characterized by a mirror-symmetric gradient (i.e. high-low-high frequency gradient) in the Heschl's region (see Figs. 3 and 4). This result is in agreement with previous functional results obtained using GE-EPI both at $3 \mathrm{~T}$ (Humphries et al., 2010; Striem-Amit et al., 2011) and $7 \mathrm{~T}$ (Formisano et al., 2003; Da Costa et al., 2011). Furthermore, using SE fMRI we found voice selective regions along the STS/STG bilaterally (see Figs. 3 and 4), a result that confirms, in single subjects and in the group, previous GE data reports (Belin et al., 2000).

While $B_{1}$ shimming could be used to measure SE-functional responses in any given functional area, the specific application to 

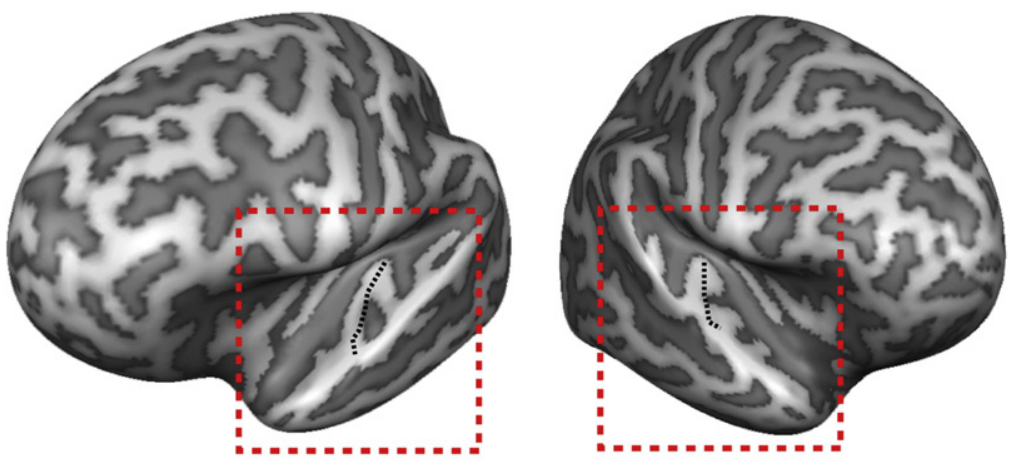

\section{Tonotopy}
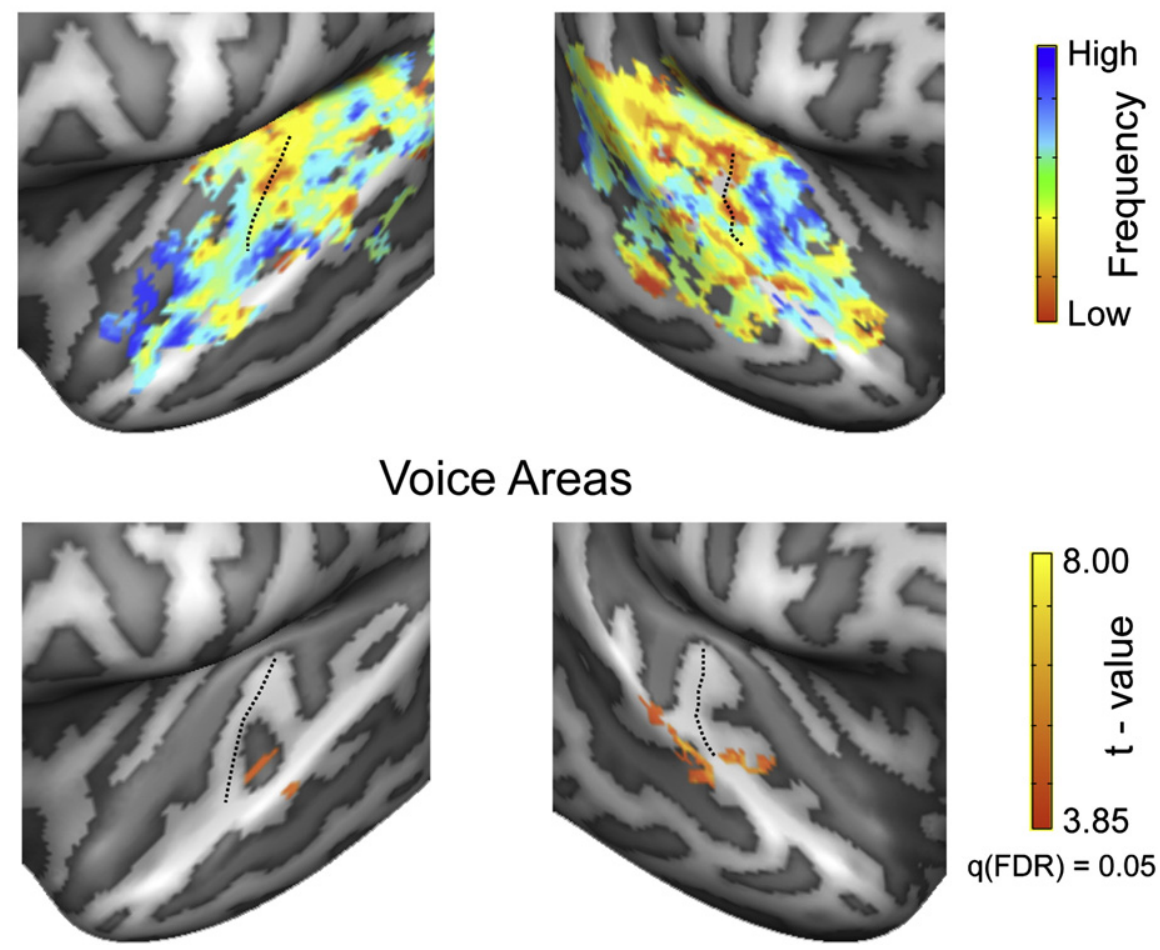

Voice Areas

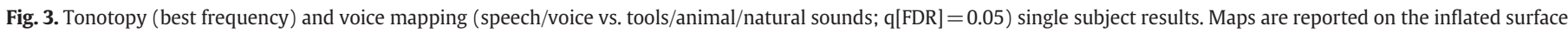
reconstruction of both hemispheres of the same subject. A black dashed line outlines the Heschl's gyrus.

auditory neuroscience could be beneficial in multiple respects. First, the impact of large draining veins on the large-scale functional organizations, such as tonotopy, could be investigated. While, a careful comparison between GE and SE fMRI is outside the scope of this paper and would require the acquisition of both data types in each subject, our single subject level and group level results are in line with previous GE fMRI reports. This could indicate that the specificity of the GE BOLD response, over a large scale functional organization in the auditory cortex, is not significantly affected by large draining veins. This is not surprising given the expected point spread function of the GE BOLD response at low or high fields (Engel et al., 1997; Shmuel et al., 2007) and the spatial scale of tonotopic organization in the auditory cortex. However, more studies would be required to answer this question quantitatively. Second and more importantly, designing an efficient strategy to measure $\mathrm{T}_{2}$ weighted functional responses with high sensitivity, as demonstrated in the present study, will permit higher resolution acquisitions. This will allow for investigating the microscopic functional architecture of the auditory cortex (i.e. cortical columns and cortical layers) with high degrees of spatial accuracy, as was shown in visual areas.

\section{Conclusions}

We demonstrated that by combining a multi-channel transmit RF coil with $\mathrm{B}_{1}$ shimming methods, high quality highly efficient multi-slice $\mathrm{T}_{2}$-weighted SE-EPI fMRI can be obtained in the bilateral auditory cortex of the human brain at $7 \mathrm{~T}$. With this approach, reliable functional BOLD responses were obtained for both synthetic tones and complex natural sounds. Maps of large-scale auditory organization, such as tonotopy and voice selective regions, derived from SE-EPI fMRI were consistent with previous GE-EPI fMRI 7 T studies. Future studies will aim to investigate finer scale functional organizations in the auditory cortex.

\section{Acknowledgments}

This work was supported in part by the National Institutes of Health (R01 EB000331, R21 EB009133, R44NS063537, P30 NS057091, P41 RR08079) and the W.M. Keck Foundation. The $7 \mathrm{~T}$ magnet purchase was funded in part by NSF DBI-9907842 and NIH S10 RR1395. 

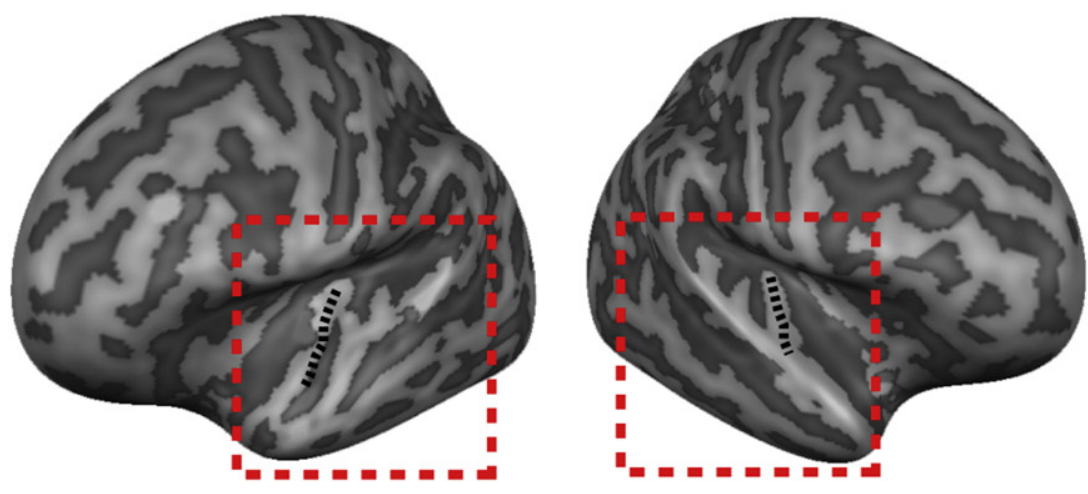

\section{Tonotopy}
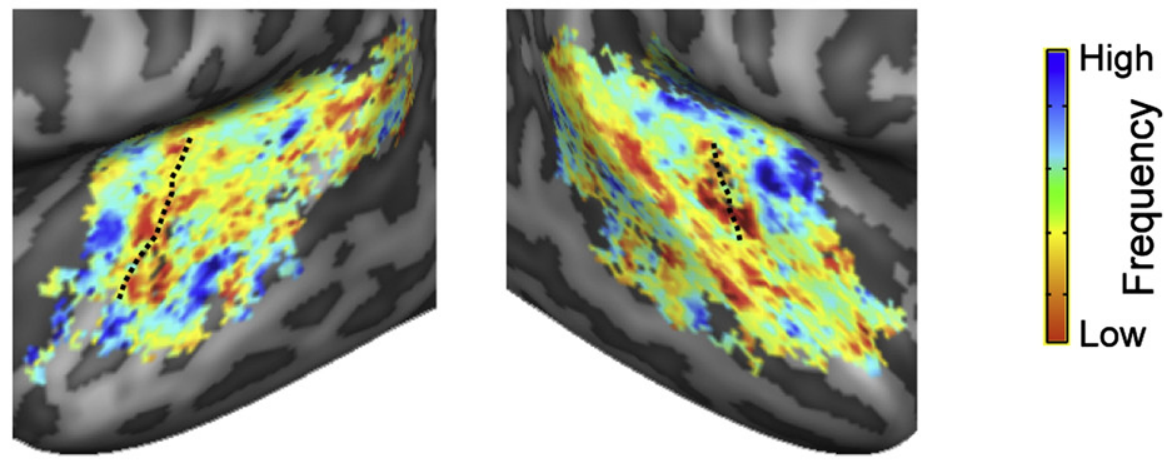

\section{Voice Areas}
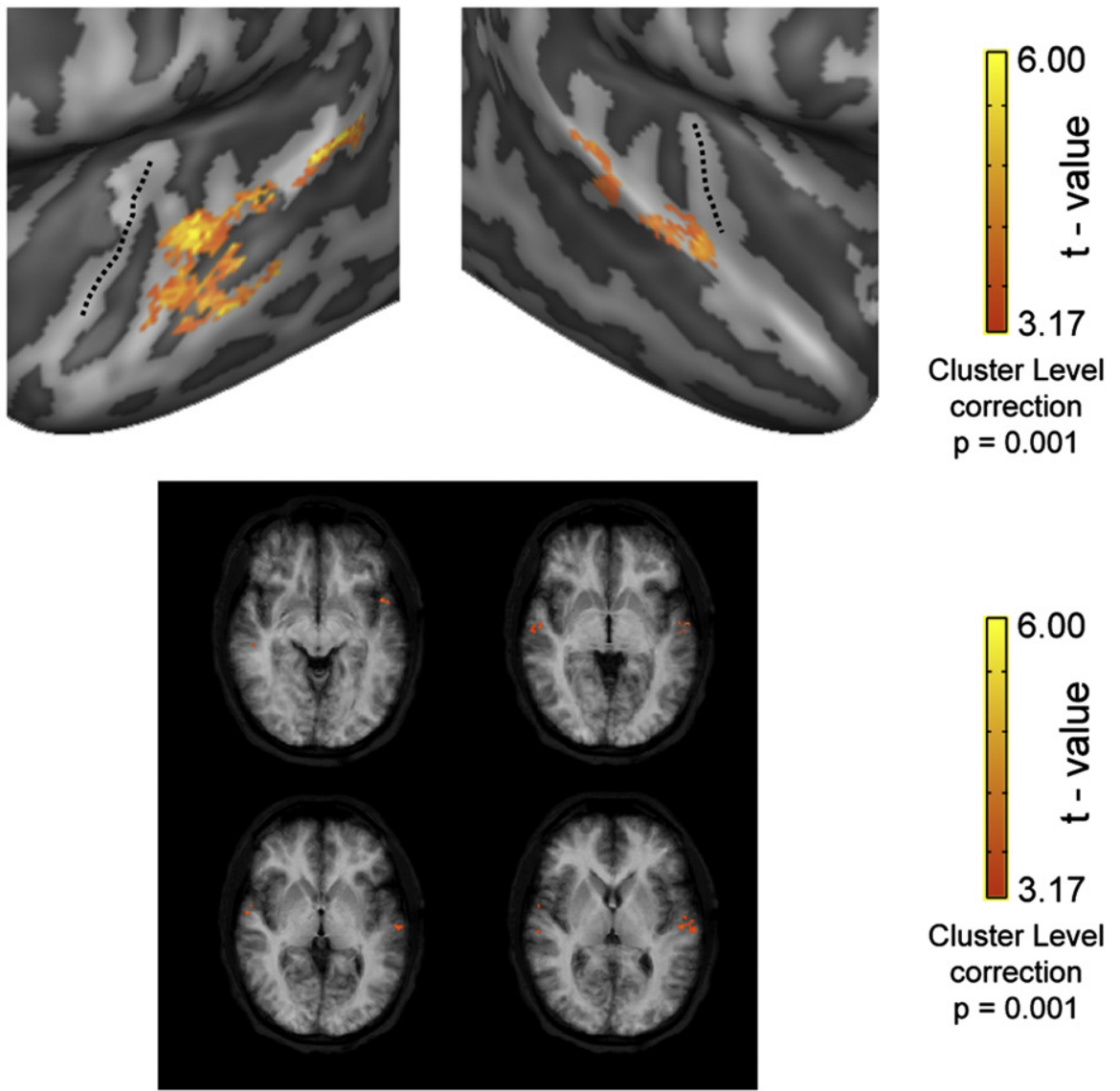

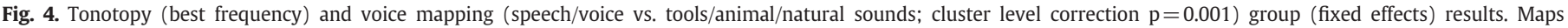

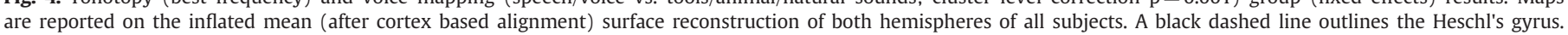
Voice mapping results are also reported superimposed to four transversal slices of the mean anatomical data (after Talairach alignment) of all subjects. 


\section{References}

Adriany, G., van De Moortele, P.-F., Wiesinger, F., Moeller, S., Strupp, J.P., Andersen, P., Snyder, C.J., Zhang, X., Chen, W., Pruessmann, K.P., Boesiger, P., Vaughan, J.T., Ugurbil, K., 2005. Transmit and receive transmission line arrays for 7 Tesla parallel imaging. Magn. Reson. Med. 53 (2), 434-445.

Adriany, G., Van de Moortele, P.-F., Ritter, J., Moeller, S., Auerbach, E.J., Akgun, C., Snyder, C.J., Vaughan, T., Ugurbil, K., 2008. A geometrically adjustable 16-channel transmit/receive transmission line array for improved RF efficiency and parallel imaging performance at 7 Tesla. Magn. Reson. Med. 59 (3), 590-597.

Bandettini, P.A., Wong, E.C., Hinks, R.S., Tikofsky, R.S., Hyde, J.S., 1992. Time course EPI of human brain function during task activation. Magn. Reson. Med. 25 (2), 390-397.

Bandettini, P., Bowtell, R., Jezzard, P., 2011. Ultrahigh field systems and applications at $7 \mathrm{~T}$ and beyond: progress, pitfalls, and potential - Bandettini - 2011 - Magnetic Resonance in Medicine - Wiley Online Library. Magnetic Resonance.

Belin, P., Zatorre, R.J., Lafaille, P., Ahad, P., Pike, B., 2000. Voice-selective areas in human auditory cortex. Nature 403 (6767), 309-312.

Cheng, K., Waggoner, R.A., Tanaka, K., 2001. Human ocular dominance columns as revealed by high-field functional magnetic resonance imaging. Neuron 32 (2), 359-374.

Da Costa, S., van der Zwaag, W., Marques, J.P., Frackowiak, R.S.J., Clarke, S., Saenz, M., 2011. Human primary auditory cortex follows the shape of Heschl's gyrus. J. Neurosci. 31 (40), 14067-14075.

Duong, T.Q., Yacoub, E., Adriany, G., Hu, X., Ugurbil, K., Kim, S.-G., 2003. Microvascular BOLD contribution at 4 and $7 \mathrm{~T}$ in the human brain: gradient-echo and spin-echo fMRI with suppression of blood effects. Magn. Reson. Med. 49 (6), 1019-1027.

Ellermann, J., Goerke, U., Morgan, P., Ugurbil, K., Tian, J., Schmitter, S., Vaughan, T., van de Moortele, P.F., 2012. Simultaneous bilateral hip joint imaging at 7 Tesla using fast transmit B(1) shimming methods and multichannel transmission - a feasibility study. NMR in biomedicine.

Engel, S.A., Glover, G.H., Wandell, B.A., 1997. Retinotopic organization in human visual cortex and the spatial precision of functional MRI. Cereb. Cortex 7 (2), 181-192.

Forman, S.D., Cohen, J.D., Fitzgerald, M., Eddy, W.F., Mintun, M.A., Noll, D.C., 1995. Improved assessment of significant activation in functional magnetic resonance imaging (fMRI): use of a cluster-size threshold. Magn. Reson. Med. 33 (5), 636-647.

Formisano, E., Kim, D.S., Di Salle, F., van De Moortele, P.-F., Ugurbil, K., Goebel, R., 2003. Mirror-symmetric tonotopic maps in human primary auditory cortex. Neuron 40 (4), 859-869.

Genovese, C.R., Lazar, N.A., Nichols, T., 2002. Thresholding of statistical maps in functional neuroimaging using the false discovery rate. Neuroimage 15 (4), 870-878.

Goebel, R., Esposito, F., Formisano, E., 2006. Analysis of functional image analysis contest (FIAC) data with brainvoyager QX: from single-subject to cortically aligned group general linear model analysis and self-organizing group independent component analysis. Hum. Brain Mapp. 27 (5), 392-401.

Harmer, J., Sanchez-Panchuelo, R.M., Bowtell, R., Francis, S.T., 2011. Spatial location and strength of BOLD activation in high-spatial-resolution fMRI of the motor cortex: a comparison of spin echo and gradient echo fMRI at 7 T. NMR in biomedicine.

Humphries, C., Liebenthal, E., Binder, J.R., 2010. Tonotopic organization of human auditory cortex. Neuroimage 50 (3), 1202-1211.

Kwong, K.K., Belliveau, J.W., Chesler, D.A., Goldberg, I.E., Weisskoff, R.M., Poncelet, B.P., Kennedy, D.N., Hoppel, B.E., Cohen, M.S., Turner, R., 1992. Dynamic magnetic resonance imaging of human brain activity during primary sensory stimulation. Proc. Natl. Acad. Sci. U. S. A. 89 (12), 5675-5679.

Lee, S.P., Silva, A.C., Ugurbil, K., Kim, S.G., 1999. Diffusion-weighted spin-echo fMRI at 9.4 T: microvascular/tissue contribution to BOLD signal changes. Magn. Reson. Med. 42 (5), 919-928.

Menon, R.S., Ogawa, S., Strupp, J.P., Ugurbil, K., 1997. Ocular dominance in human V1 demonstrated by functional magnetic resonance imaging. J. Neurophysiol. 77 (5), 2780-2787.

Metzger, G.J., Snyder, C., Akgun, C., Vaughan, T., Ugurbil, K., Van de Moortele, P.-F., 2008. Local $\mathrm{B}_{1}^{+}$shimming for prostate imaging with transceiver arrays at $7 \mathrm{~T}$ based on subject-dependent transmit phase measurements. Magn. Reson. Med. 59 (2), 396-409.

Metzger, G.J., van de Moortele, P.F., Akgun, C., Snyder, C.J., Moeller, S., Strupp, J. Andersen, P., Shrivastava, D., Vaughan, T., Ugurbil, K., Adriany, G., 2010. Performance of external and internal coil configurations for prostate investigations at 7 T. Magn. Reson. Med. 64 (6), 1625-1639.

Metzger, G.J., DelaBarre, L., Bi, X., Shab, S., Zuehlsdorff, S., Vaughan, T.J., Ugurbil, K., 2011. Left coronary artery imaging at $7 \mathrm{~T}$ : initial results using multiple $\mathrm{B}_{1}^{+}$shimming algorithms and targets. Proc. Intl. Soc. Mag. Reson. Med. 1.
Metzger, G.J., Auerbach, E.J., Akgun, C., Simonson, J., Bi, X., Ugurbil, K., van de Moortele, P.F 2012. Dynamically applied $B_{1}$ shimming solutions for non-contrast enhanced renal angiography at 7.0 Tesla. Magn. Reson. Med. http://dx.doi.org/10.1002/mrm.24237.

Norris, D.G., 2012. Spin-Echo fMRI: the poor relation? Neuroimage 62 (2), 1109-1115 (Aug 15).

Ogawa, S., Tank, D.W., Menon, R.S., Ellermann, J.M., Kim, S.-G., Merkle, H., Ugurbil, K., 1992. Intrinsic signal changes accompanying sensory stimulation: functional brain mapping with magnetic resonance imaging. Proc. Natl. Acad. Sci. U. S. A. 89 (13), 5951-5955.

Schmitter, S., Adriany, G., Auerbach, E.J., Ugurbil, K., van de Moortele, P.F., 2012. Neither flat profile nor black spots: a simple method to achieve acceptable CP-like mode transmit B1 pattern for whole brain imaging with transmit arrays at 7 Tesla. Proc. Int. Soc. Mag. Reson. Med. (3472).

Schönwiesner, M., Zatorre, R.J., 2009. Spectro-temporal modulation transfer function of single voxels in the human auditory cortex measured with high-resolution fMRI. Proc. Natl. Acad. Sci. U. S. A. 106 (34), 14611-14616.

Shmuel, A., Yacoub, E., Chaimow, D., Logothetis, N.K., Ugurbil, K., 2007. Spatio-temporal point-spread function of fMRI signal in human gray matter at 7 Tesla. Neuroimage 35 (2), 539-552.

Stollberger, R., Wach, P., 1996. Imaging of the active B1 field in vivo. Magn. Reson. Med. 35 (2), 246-251.

Striem-Amit, E., Hertz, U., Amedi, A., 2011. Extensive cochleotopic mapping of human auditory cortical fields obtained with phase-encoding fMRI. PLoS One 6 (3), e17832.

Sun, P., Ueno, K., Waggoner, R.A., Gardner, J.L., Tanaka, K., Cheng, K., 2007. A temporal frequency-dependent functional architecture in human V1 revealed by highresolution fMRI. Nat. Neurosci. 10 (11), 1404-1406.

Ugurbil, K., Adriany, G., Andersen, P., Chen, W., Garwood, M., Gruetter, R., Henry, P.-G. Kim, S.-G., Lieu, H., Tkac, I., Vaughan, J.T., van De Moortele, P.-F., Yacoub, E., Zhu, X.-H., 2003. Ultrahigh field magnetic resonance imaging and spectroscopy. Magn. Reson. Imaging 21 (10), 1263-1281.

Uğurbil, K., Toth, L., Kim, D.S., 2003. How accurate is magnetic resonance imaging of brain function? Trends Neurosci. 26 (2), 108-114.

Ugurbil, K., Chen, W., Harel, N., van de Moortele, P.F., Yacoub, E., Zhu, X.-H., Uludag, K. 2006. Magnetic resonance imaging of brain function. In: Akay, M. (Ed.), Wiley Encyclopedia of Biomedical Engineering. Hoboken: John Wiley \& Sons, Inc., Hoboken, pp. 647-668.

van de Moortele, P.F., Ugurbil, K., 2009. Very fast multi channel $B_{1}$ calibration at high field in the small flip angle regime. Proc. Intl. Soc. Mag. Reson. Med. 1.

van De Moortele, P.-F., Akgun, C., Adriany, G., Moeller, S., Ritter, J., Collins, C.M., Smith, M.B., Vaughan, J.T., Ugurbil, K., 2005. B(1) destructive interferences and spatial phase patterns at $7 \mathrm{~T}$ with a head transceiver array coil. Magn. Reson. Med. 54 (6), 1503-1518.

van de Moortele, P.F., Snyder, C.J., DelaBarre, L., Adriany, G., Vaughan, J.T., Ugurbil, K., 2007. Calibration tools for RF shim at very high field with multiple element RF coils: from ultra fast local relative phase to absolute magnitude $B_{1}$ mapping Proc. Intl. Soc. Mag. Reson. Med. (1676).

Vaughan, J.T., Garwood, M., Collins, C.M., Liu, W., DelaBarre, L., Adriany, G., Andersen, P., Merkle, H., Goebel, R., Smith, M.B., Ugurbil, K., 2001. 7 T vs. 4 T: RF power, homogeneity, and signal-to-noise comparison in head images. Magn. Reson. Med. 46 (1), 24-30.

Yacoub, E., Shmuel, A., Pfeuffer, J., van De Moortele, P.-F., Adriany, G., Andersen, P., Vaughan, J.T., Merkle, H., Ugurbil, K., Hu, X., 2001. Imaging brain function in humans at 7 Tesla. Magn. Reson. Med. 45 (4), 588-594.

Yacoub, E., Duong, T.Q., van De Moortele, P.-F., Lindquist, M., Adriany, G., Kim, S.-G. Ugurbil, K., Hu, X., 2003. Spin-echo fMRI in humans using high spatial resolutions and high magnetic fields. Magn. Reson. Med. 49 (4), 655-664.

Yacoub, E., van De Moortele, P.-F., Shmuel, A., Ugurbil, K., 2005. Signal and noise characteristics of Hahn SE and GE BOLD fMRI at $7 \mathrm{~T}$ in humans. Neuroimage 24 (3), 738-750.

Yacoub, E., Shmuel, A., Logothetis, N., Ugurbil, K., 2007. Robust detection of ocular dominance columns in humans using Hahn Spin Echo BOLD functional MRI at 7 Tesla. Neuroimage 37 (4), 1161-1177.

Yacoub, E., Harel, N., Ugurbil, K., 2008. High-field fMRI unveils orientation columns in humans. Proc. Natl. Acad. Sci. U. S. A. 105 (30), 10607-10612.

Yarnykh, V.L., 2007. Actual flip-angle imaging in the pulsed steady state: a method for rapid three-dimensional mapping of the transmitted radiofrequency field. Magn. Reson. Med. 57 (1), 192-200.

Zimmermann, J., Goebel, R., De Martino, F., Van de Moortele, P.-F., Feinberg, D., Adriany, G., Chaimow, D., Shmuel, A., Ugurbil, K., Yacoub, E., 2011. Mapping the organization of axis of motion selective features in human area MT using high-field fMRI. PLoS One 6 (12), e28716. 